\title{
Multiscale Transparent Electrode Architecture for Efficient Light Management and Carrier Collection in Solar Cells
}

\author{
Mathieu Boccard,* Corsin Battaglia, Simon Hänni, Karin Söderström, Jordi Escarré, Sylvain Nicolay, \\ Fanny Meillaud, Matthieu Despeisse, and Christophe Ballif
}

Institute of Microengineering (IMT), Photovoltaics and Thin Film Electronics Laboratory, Ecole Polytechnique Fédérale de Lausanne (EPFL), Rue A.-L. Breguet 2, CH-2000 Neuchâtel, Switzerland

\section{Supporting Information}

ABSTRACT: The challenge for all photovoltaic technologies is to maximize light absorption, to convert photons with minimal losses into electric charges, and to efficiently extract them to the electrical circuit. For thin-film solar cells, all these tasks rely heavily on the transparent front electrode. Here we present a multiscale electrode architecture that allows us to achieve efficiencies as high as $14.1 \%$ with a thin-film silicon tandem solar cell employing only $3 \mu \mathrm{m}$ of silicon. Our approach combines the versatility of nanoimprint lithography, the unusually high carrier mobility of hydrogenated indium oxide (over $100 \mathrm{~cm}^{2} / \mathrm{V} / \mathrm{s}$ ), and the unequaled lightscattering properties of self-textured zinc oxide. A multiscale texture provides light trapping over a broad wavelength range while ensuring an

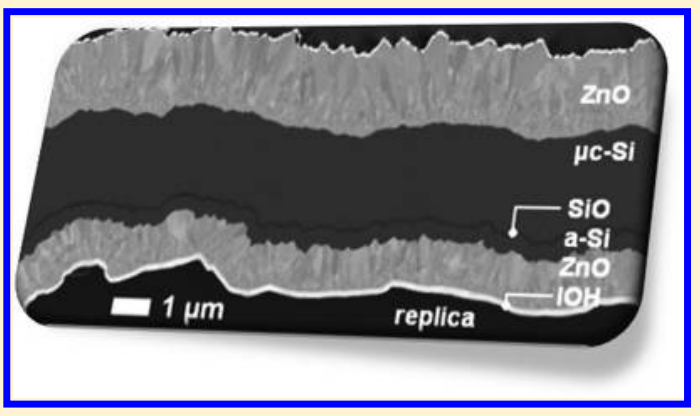
optimum morphology for the growth of high-quality silicon layers. A conductive bilayer stack guarantees carrier extraction while minimizing parasitic absorption losses. The tunability accessible through such multiscale electrode architecture offers unprecedented possibilities to address the trade-off between cell optical and electrical performance.

KEYWORDS: Photovoltaics, thin-film silicon solar cells, texture, nanoimprinting, light trapping

$\mathrm{T}$ he power of the sun ( $86 \mathrm{PW}$ on the Earth's surface $)^{1}$ has the potential to solve the energy crisis of the century. By converting sunlight directly to electricity, photovoltaics has a major role to play in the energy mix of the near future, provided that the requirements of high efficiency and low cost are met simultaneously. Thin-film technologies have huge potential due to their proven low production costs ${ }^{2,3}$ and their continuous efficiency increase. Fully exploiting the efficiency potential of a solar cell necessitates maximizing absorption of photons, converting them with minimal losses to electric charges, and efficiently extracting those charges to the electrical circuit. Accomplishing in an optimum way these three tasks is a common struggle faced by all photovoltaic technologies. ${ }^{4-6}$

We focus here on the transparent front electrode, which is a key element in fulfilling these three tasks. As it is the first layer of the device crossed by the incoming light, its transparency is fundamental. At the same time, it has to be sufficiently conductive to extract carriers without significant resistive losses. As conductivity is improved, transparency tends to decrease requiring a compromise to be found. ${ }^{6-12}$

In addition, especially for thin-film technologies, the front electrode has to couple light efficiently into the absorber layer and provide strong light trapping. ${ }^{11-18}$ As it also serves as substrate for cell deposition, its morphology must be suitable for high-quality absorber material growth: Rugged electrode morphologies acclaimed for optimum light management generally trigger the formation of spatially inhomogeneous and porous areas in the photoactive layer. These defective areas degrade the cell performance by lowering the potential energy of the electric charges created by absorbed light. ${ }^{17-20}$

The double trade-off on one hand between transparency and conductivity and on the other hand strong light trapping and high-quality absorber material is traditionally addressed by a single transparent conductive oxide (TCO) layer. In this letter we propose an innovative electrode architecture composed of three functional layers, which allows us to decouple these tradeoffs and address them separately. By combining nanoimprint lithography and self-textured zinc oxide $(\mathrm{ZnO})$, we obtain a multiscale texture that enables optimal light coupling and strong light trapping over a wide spectral range while providing ideal electrode morphology for high-quality film growth. By furthermore including a high-mobility hydrogenated indium oxide $(\mathrm{IOH})$ layer in the stack, excellent transparency can be achieved while maintaining sufficient conduction for efficient carrier extraction. We validate our approach by demonstrating a thin-film silicon tandem solar cell with an absorber layer thickness of only $3 \mu \mathrm{m}$ and an excellent initial efficiency of $14.1 \%$, which lies among the highest reported values for this technology. ${ }^{21,22}$

The thin-film silicon approach is very attractive due to its particularly low production costs ${ }^{2}$ and use of stable, nontoxic, and abundant materials. ${ }^{23}$ We focus here on the Micromorph

Received: November 7, 2011

Revised: January 31, 2012 
concept, ${ }^{6}$ which consists of a stack of hydrogenated amorphous silicon and hydrogenated microcrystalline silicon (a-Si and $\mu \mathrm{c}$ $\mathrm{Si}$ ) subcells. This tandem device structure has an efficiency potential of over $30 \%$ thanks to its ideal combination of band gaps: ${ }^{24}$ a-Si efficiently absorbs visible light (of energy higher than its band gap of approximately $1.7 \mathrm{eV}$ ), while $\mu \mathrm{c}-\mathrm{Si}$ absorbs some infrared light (down to $1.1 \mathrm{eV}$ ). Both materials have a low absorption coefficient close to their band gap, requiring large material thicknesses (typically a few micrometers and a few hundred micrometers, respectively) to absorb most of the light. However, due to their short carrier diffusion lengths, especially after light-induced degradation, ${ }^{25}$ the thicknesses are limited to a few hundreds and a few thousands of nanometers for, respectively, a-Si and $\mu \mathrm{c}-\mathrm{Si}$ subcells. ${ }^{26,27}$ As the subcells are connected in series, the current flowing out of the Micromorph device will be limited by the subcell generating the lowest current. The transparent electrode substrate must therefore provide light management for both subcells, i.e., in a broad range of wavelengths $(400-1100 \mathrm{~nm})$. Also, the substrate morphology must be suitable for growing both high-quality a-Si and $\mu \mathrm{c}-\mathrm{Si}$ material.

Figure 1 is a graphical representation of Micromorph device properties as a function of two main morphological character-

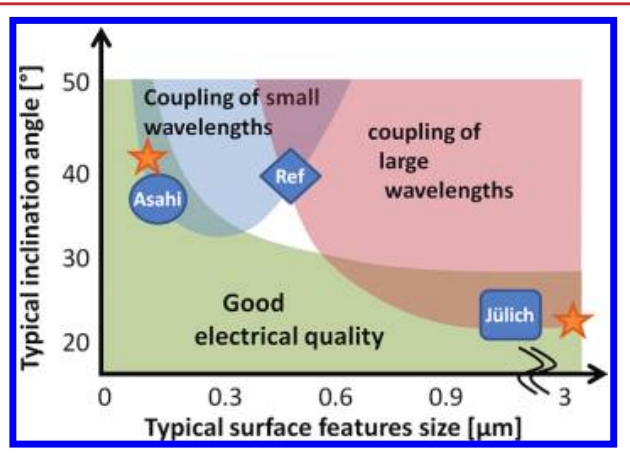

Figure 1. Schematic distribution of Micromorph device characteristics as a function of two main morphological properties of the substrate: the typical size of the features composing its surface and their typical inclination angle. Three state-of-the-art TCO substrates are represented: tin oxide (Asahi), sputter-etched $\mathrm{ZnO}$ (Jülich), and inhouse LPCVD $\mathrm{ZnO}$ (ref). The stars correspond to the multiscale textured substrate.

istics of the substrate: The typical size of the features composing its surface, and their typical inclination angle (characterizing the sharpness of the features). Three commonly used rough TCO-based substrates are represented: textured tin oxide (Asahi) ${ }^{28}$ sputter-etched zinc oxide (Jülich), ${ }^{11}$ and an inhouse $\mathrm{ZnO}$ layer deposited by low-pressure chemical vapor deposition (LPCVD) optimized for Micromorph devices (ref). ${ }^{29}$

Three main areas are sketched, corresponding to substrate morphologies favoring coupling of small wavelengths (requiring small and sharp features), scattering of large wavelengths (requiring large features), and good electrical quality (requiring small or smooth features). These areas are derived from numerous experimental data from our group ${ }^{29}$ (also Supporting Information), in agreement with literature. ${ }^{30}$ It is possible to combine two out of the three qualities in a single substrate, but no substrate has all three qualities. This is what we seek with the multiscale morphology corresponding to the orange stars in Figure 1, which combines small and sharp features on top of large and smooth ones. This results in a surface exhibiting a double modulation (Figure 2a), with $100 \mathrm{~nm}$-scale pyramids and $3 \mu \mathrm{m}$-scale hills. The two distinct textures are designed to

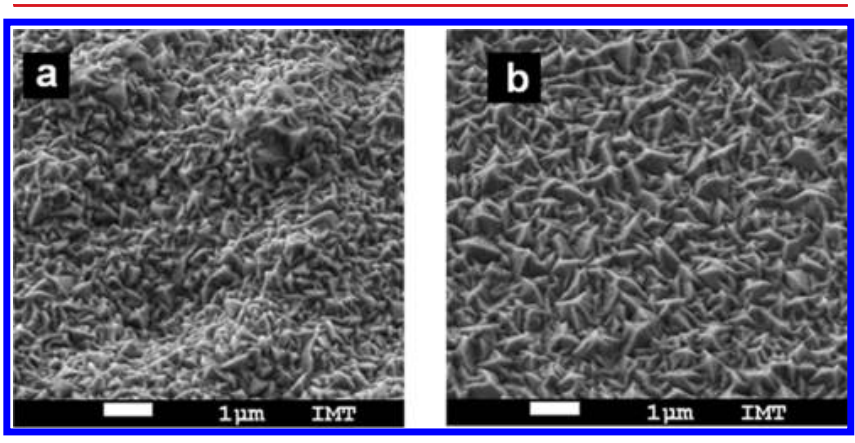

Figure 2. (a,b) SEM images of the surfaces of the multiscale textured electrode (a) and the reference $\mathrm{ZnO}$ electrode (b).

achieve excellent light management for short and large wavelengths. Also, both features are chosen to enable the growth of high-quality silicon material. ${ }^{29,31}$

Figures 3 and $4 \mathrm{a}$ show a schematic drawing and a scanning electron microscopy (SEM) image of a cross section milled

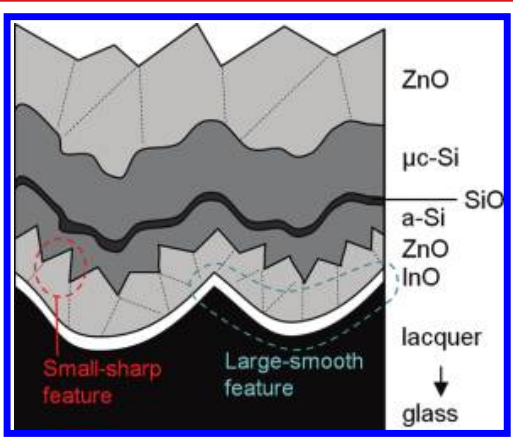

Figure 3. Schematic drawing of a cross section of a Micromorph cell deposited on a multiscale textured substrate evidencing the small-sharp and large-smooth features.

with a focused ion beam (FIB) through a Micromorph cell deposited on this multiscale textured substrate. A smooth, micrometer-scale texture is first obtained on flat glass by replication via a high-fidelity UV-nil nanoimprint lithography technique, as detailed in ref 32 . The master used here is a 16 $\mu \mathrm{m}$-thick LPCVD $\mathrm{ZnO}$ layer smoothened by a $3 \mathrm{~h}$ plasma treatment. $^{33}$ Its surface corresponds to the right-side orange star in Figure 1. Next, a $120 \mathrm{~nm}$-thick IOH layer is deposited on top by sputtering as described in ref 34. Its high mobility and low carrier density $\left(\mu_{\mathrm{H}}>100 \mathrm{~cm}^{2} / \mathrm{V} / \mathrm{s}\right.$ and $N_{\mathrm{D}}=1 \times 10^{20}$ $\mathrm{cm}^{-3}$ ) make it highly transparent for the whole wavelength range of interest for silicon solar cells for a sheet resistance $\left(R_{\mathrm{sh}}\right)$ below $50 \Omega .^{8,12}$ Finally, a $1 \mu \mathrm{m}$-thick nonintentionally doped LPCVD $\mathrm{ZnO}$ layer is deposited on top. Its $R_{\mathrm{sh}}$ of approximately $50 \Omega\left(N_{\mathrm{D}}=4 \times 10^{19}, \mu_{\mathrm{H}}=30 \mathrm{~cm}^{2} / \mathrm{V} / \mathrm{s}\right)$ makes the front electrode stack $R_{\mathrm{sh}}$ approximately $25 \Omega$, while its low carrier density keeps the electrode absorption low. This layer also offers the small but sharp features that guarantee strong light coupling into the a-Si top cell. ${ }^{29,35} \mathrm{~A}$ schematic representation of the multiscale front electrode fabrication sequence with more details is available as Supporting Information.

Micromorph cells are then grown by plasma-enhanced chemical vapor deposition (PECVD) in a dual-chamber 


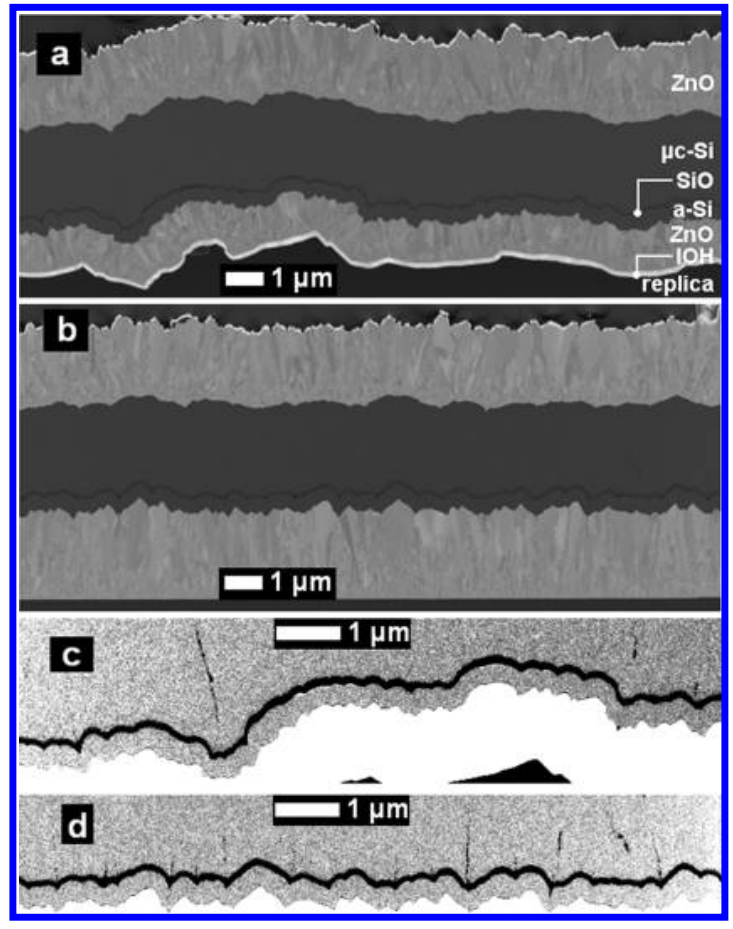

Figure 4. $(\mathrm{a}, \mathrm{b})$ SEM images of a FIB cut across a Micromorph cell deposited on a multiscale textured substrate (a) and on a reference $\mathrm{ZnO}$ layer (b). (c,d) High-contrast zoom image of (a) and (b) emphasizing bad areas (dark vertical lines) formed at the beginning of $\mu \mathrm{c}-\mathrm{Si}$ growth when pinches are present at the silicon-oxide-based intermediate reflector surface due to the roughness of the front electrode.

research-scale system. The thicknesses of the intrinsic layers of the top and bottom cells are $290 \mathrm{~nm}$ and $2.6 \mu \mathrm{m}$. A $60 \mathrm{~nm}$ thick silicon-oxide-based intermediate reflector ${ }^{36}$ is used to reflect part of the visible light back into the top cell, enabling high top cell currents for thin layers. Doped silicon-rich silicon-oxide layers, recently developed in our laboratory, are also implemented in the bottom cell to limit the influence of inhomogeneous and low-quality silicon regions on cell performance. $^{37,38}$ As can be seen in Figure 3, the a-Si cell grows directly on the small and sharp texture of the $\mathrm{ZnO}$ layer, benefiting of its light scattering ability. These sharp features (prejudicial to good quality $\mu \mathrm{c}-\mathrm{Si}$ growth) are however softened by the a-Si and $\mathrm{SiO}$ layers, leaving mostly a smooth and largescale modulation at the surface of the $\mathrm{SiO}$ intermediate layer, ideal for good quality $\mu \mathrm{c}$-Si growth and light trapping in the $\mu \mathrm{c}$ Si cell.

The same Micromorph structure is also deposited on a reference flat substrate (sample A, flat glass/IOH and a thin protective $\mathrm{ZnO}$ layer), a state-of-the-art single-layer LPCVD $\mathrm{ZnO}$ electrode of which the surface is shown in Figure $2 \mathrm{~b}$ and $\mathrm{a}$ cross section in Figure $4 \mathrm{~b}$ (sample B, flat glass $/ 2.4 \mu \mathrm{m} \mathrm{ZnO}$ ), and an electrode stack with only the smaller scale morphology (sample D, flat glass/IOH/1 $\mu \mathrm{m} \mathrm{ZnO} \mathrm{stack).} \mathrm{A} 1$ min plasma surface treatment is performed on all substrates before silicon deposition. A 4 min treatment is also applied to the state-ofthe-art $\mathrm{ZnO}$ layer to reduce the typical inclination of the surface and make it more suitable for high-quality silicon deposition. ${ }^{29,33}$

For all samples, the back contact is a lightly doped $2.4 \mu \mathrm{m}$ thick LPCVD ZnO layer, and a white dielectric reflector is applied at the back of each cell.
Figure 5 presents current density-voltage $(J(V))$ characteristics of the cells deposited on substrates $A-C$, measured with a

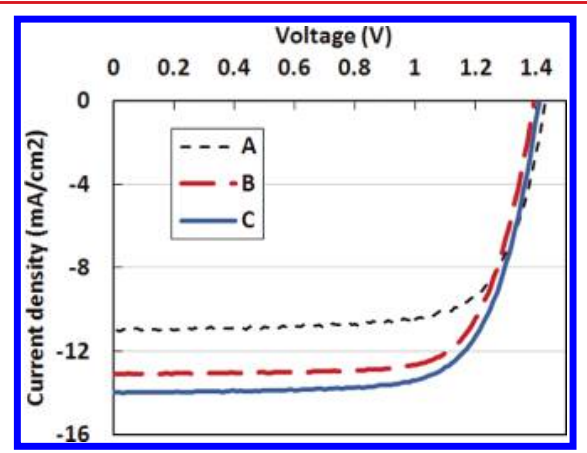

Figure 5. $J(V)$ curves of Micromorph cells deposited on a flat substrate (A), on a reference $\mathrm{ZnO}$ electrode (B), and on a multiscale textured substrate $(\mathrm{C})$.

dual lamp sun simulator in standard test conditions $\left(25{ }^{\circ} \mathrm{C}\right.$, global air mass 1.5 (AM1.5 g) spectrum, $1000 \mathrm{~W} / \mathrm{m}^{2}$ ). Table 1 summarizes their open-circuit voltages $\left(V_{\mathrm{OC}}\right)$, fill factors $(\mathrm{FF})$, short circuit current densities $\left(J_{\mathrm{SC}}\right)$, and efficiencies. $V_{\mathrm{OC}}$ and FF are calculated from the $J(V)$ characteristics of the cells, and $J_{\text {sc }}$ of the top and bottom subcells is determined by convolution of the external quantum efficiency (EQE) and the incoming photon flux of the AM1.5 g spectrum.

Table 1 shows that the highest initial efficiency of $14.1 \%$ is obtained on the multiscale substrate, which is a $2.8 \%$ absolute gain compared to the flat substrate and a $0.9 \%$ gain compared to the single-layer reference $\mathrm{ZnO}$ substrate. As can be seen in Figure 5, the efficiency gain is obtained through both $J_{S C}$ and $V_{\text {OC }}$ increases. This suggests that our approach allows for both better material quality and efficient light management.

Concerning the $J_{\mathrm{SC}}$ values, it can be seen that high top cell currents $\left(>14 \mathrm{~mA} / \mathrm{cm}^{2}\right)$ can be obtained on all rough substrates, due to the particularly suitable morphology of LPCVD ZnO. However, a high bottom cell current is only achieved on the multiscale textured substrate. This can clearly be attributed to better light trapping than in samples A and D, where no large-scale texture is present to provide light scattering of large wavelengths. The comparison to the reference $\mathrm{ZnO}$ substrate is less straightforward, as discussed in the following. Figure 6 presents the EQE of the top and bottom subcells of devices $\mathrm{A}-\mathrm{C}$ as well as total device absorption [represented as one minus the reflection from the device $(1-R)]$ for devices $\mathrm{B}$ and $\mathrm{C}$.

One first notices that part of the current gain in the bottom cell for sample C compared to sample B is obtained in the $550-750 \mathrm{~nm}$ range. This is accompanied by a loss in the top cell and thus does not represent improved light trapping. Another striking point is that more light is actually coming out of the device on the multiscale textured electrode than on the reference $\mathrm{ZnO}$ substrate. As the EQE curve is higher for the multiscale textured electrode, this indicates a reduction in parasitic absorption $\left(A_{\mathrm{P}}\right)^{7} . A_{\mathrm{P}}$ comes mostly from doped layers, electrodes, and back reflector in our devices. ${ }^{7,34}$ As the doped layers, back electrode, and back reflector are the same in all cases, this difference can be attributed to better transparency of our multiscale textured electrode. Thus, the multiscale substrate reaches the highest summed current of the series mostly because it has better transparency, yet maintains excellent light trapping qualities. 
Table 1. Characteristics of $1 \mathrm{~cm}^{2}$ Micromorph Cells Grown on Various Substrates

\begin{tabular}{lcccccc} 
& $V_{\mathrm{OC}}(\mathrm{V})$ & $\mathrm{FF}(\%)$ & $J_{\mathrm{SC}}$, top $\left(\mathrm{mA} / \mathrm{cm}^{2}\right)$ & $J_{\mathrm{SC}}$, bottom $\left(\mathrm{mA} / \mathrm{cm}^{2}\right)$ & $J_{S C}$, sum $\left(\mathrm{mA} / \mathrm{cm}^{2}\right)$ & $\mathrm{Eff}(\%)$ \\
(A) flat IOH & 1.432 & 71.8 & 12.6 & 11.0 & 13.1 & 23.6 \\
(B) ref LPCVD ZnO & 1.395 & 72.4 & 14.8 & 14.0 & 27.9 & 13.2 \\
(C) multiscale texture & 1.411 & 71.5 & 14.3 & 12.6 & 28.3 \\
(D) IOH + thin LPCVD ZnO & 1.420 & 75.0 & 14.5 & 27.1 & 13.1 \\
\hline
\end{tabular}

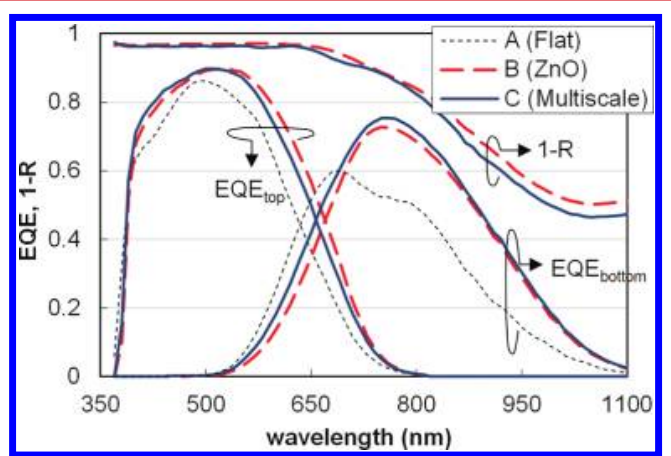

Figure 6. EQE curves of top and bottom subcells of Micromorph cells deposited on a flat substrate (A), a reference $\mathrm{ZnO}$ substrate (B), and a multiscale textured substrate $(\mathrm{C})$. Total device absorption (corresponding to $1-R)$ is also plotted for $\mathrm{B}$ and $\mathrm{C}(1-R$ is omitted for $\mathrm{A}$ for a better clarity).

Turning now to the $V_{\mathrm{OC}}$, which is sensitive to the electrical quality of the junctions, the highest value is achieved on the flat substrate (A), as expected from Figure 1. For the reference $\mathrm{ZnO}$ substrate, the roughness drastically reduces the $V_{\mathrm{OC}}$ with a $37 \mathrm{mV}$ loss caused by creation of defective areas. With the multiscale texture, the loss is reduced to only $21 \mathrm{mV}$, indicating a more homogeneous material. This correlates well with the reduced number of cracks in the $\mu \mathrm{c}$-Si layer (appearing as dark lines in the cross sections in Figure 4c,d). The sharp $\mathrm{ZnO}$ features, mostly responsible for these cracks, are more efficiently smoothened out by the a-Si cell in the multiscale texture case than in the single-scale case, as a thinner $\mathrm{ZnO}$ layer (resulting in smaller features) can be used. The reductions of sheet conductance and light scattering at large wavelength are indeed compensated by, respectively, $\mathrm{IOH}$ and the large modulation of the replica, reproduced at the $\mathrm{ZnO}-\mathrm{Si}$ interface. From the $V_{\text {OC }}$ value observed on sample $\mathrm{D}$, we can deduce that around $10 \mathrm{mV}$ are still to be recovered by improving the small and large features.

Finally, the FF of tandem devices is a delicate parameter as it is strongly influenced by the difference between the subcell $J_{\mathrm{SC}} \mathrm{S}$ (also called mismatch). ${ }^{29,39}$ A detailed analysis of the FF changes in tandem devices is outside the scope of this work, but a few hints about our particular case follow. The lowest FF of the series is obtained for sample $\mathrm{C}$, which also has the closest subcells currents. Indeed, FF gains of up to $2 \%$ per $\mathrm{mA} / \mathrm{cm}^{2}$ of mismatch are reported. ${ }^{29,39}$ This explains a large part of the $3.5 \%$ FF drop between samples $\mathrm{D}$ and $\mathrm{C}$, where the introduction of large-scale features generates a $1.6 \mathrm{~mA} / \mathrm{cm}^{2}$ mismatch reduction. The FF loss between the reference $\mathrm{ZnO}$ and the multiscale textured electrode can also be attributed to mismatch reduction ( 0.3 compared to $\left.1.7 \mathrm{~mA} / \mathrm{cm}^{2}\right)$. Indeed, observing only $1 \%$ FF loss for $1.4 \mathrm{~mA} / \mathrm{cm}^{2}$ mismatch reduction suggests that better quality material is grown on the multiscale substrate. This correlates well with the $V_{\mathrm{OC}}$ trend and the crack densities observed in the SEM cross sections in Figure 4c,d. Thus, unlike in our earlier reports, ${ }^{12,40}$ no FF losses are present due to degradation of material electrical quality or high sheet resistances. Both issues previously faced when using nanoimprinted front electrodes are solved, with the multiscale texture and the two-layer conductive stack.

As cell efficiency is the ultimate metric, our approach exhibits strong potential by showing $14.1 \%$ initial efficiency for a thinfilm silicon tandem device with only $3 \mu \mathrm{m}$ of absorber material. Importantly, all processes involved are compatible with largearea industrial production. This approach significantly improves flexibility when addressing the compromise between strong light scattering and high-quality material growth. It also opens new roads for light harvesting in solar cells, by splitting the spectrally wide requirements into several different dedicated photonic structures. While we restricted our study to the morphologies accessible with LPCVD $\mathrm{ZnO}$, many other photonic structure combinations are possible and easily accessible thanks to the versatility of nanoimprinting that already prove many possibilities for photovoltaics applications. $12,40-42$

In conclusion, we proposed here a new architecture for thinfilm solar cells substrates that enabled very high efficiencies to be reached with thin silicon layers. We revealed that the front electrode has numerous conflicting tasks to perform and that they can be split by using several dedicated layers. We suggested an innovative design combining three layers, each one dedicated to a specific task. A smooth and large-scale texture, fabricated in lacquer by nanoimprint lithography, ensures scattering of near-infrared light for the bottom cell while preserving a morphology suitable for high-quality silicon growth. A subsequent $1 \mu$ m-thick, highly transparent LPCVD $\mathrm{ZnO}$ layer provides small and sharp features, guaranteeing coupling of ultraviolet and visible light into the top cell, again with minimal impact on silicon growth. Finally, a thin, highmobility hydrogenated indium oxide layer ensures sufficient conductance for electrical carrier extraction without compromising transparency. Compared to state-of-the-art substrates, this new approach demonstrated similar light scattering properties together with higher transparency and better suitability for high-quality silicon growth. A noteworthy $14.1 \%$ initial efficiency was achieved, and we believe that the multiscale architecture offers unprecedented possibilities for implementing innovative photonic structures in high-efficiency low-cost thin-film solar modules.

\section{ASSOCIATED CONTENT}

\section{S Supporting Information}

Maps of the characteristics $\left(V_{\mathrm{OC}}, J_{\mathrm{SC}, \text { top }}\right.$, and $J_{\mathrm{SC} \text {, bottom }}$ ) of Micromorph cells as a function of the substrate features typical size and inclination. Schematic representation and description of the multiscale front electrode fabrication sequence. This material is available free of charge via the Internet at http:// pubs.acs.org.

\section{AUTHOR INFORMATION}

\section{Corresponding Author}

*E-mail: mathieu.boccard@epfl.ch. 


\section{Notes}

The authors declare no competing financial interest.

\section{ACKNOWLEDGMENTS}

We acknowledge Mustapha Benkhaira for $\mathrm{ZnO}$ deposition, Peter Cuony and Michael Stückelberger for fruitful discussions, and Duncan Alexander for assistance with the FIB and the Swiss Federal Energy Office for funding under project 101191.

\section{REFERENCES}

(1) Hermann, W. A. Energy 2006, 31, 1685.

(2) Kratzla, T; Zindel, A.; Benz, R. Proceedings of the 5th World Conference on Photovoltaic Energy Conversion, Valencia, Spain, September 6-10, 2010; p 2807, DOI: 10.4229/25thEUPVSEC20103CO.14.1.

(3) First Solar Passes \$1 Per Watt Industry Milestone; First Solar, Inc.: Tempe, AZ; http://investor.firstsolar.com/releasedetail. $\mathrm{cfm}$ ?ReleaseID $=571539$.

(4) Tiedje, T.; Yablonovitch, E.; Cody, G. D.; Brooks, B. G. IEEE Trans. Electron Devices 1984, 31, 711.

(5) O’Reagal, B.; Grätzel, M. Nature 1991, 353, 737.

(6) Shah, A.; Torres, P.; Tscharner, R.; Wyrsch, N.; Keppner, H. Science 1999, 285, 692.

(7) Boccard, M.; Cuony, P.; Battaglia, C.; Despeisse, M.; Ballif, C. Phys. Status Solidi RRL 2010, 4-11, 326.

(8) Koida, T.; et al. Thin Solid Films 2010, 518, 2930.

(9) Gupta, A.; Compaan, A. D. Appl. Phys. Lett. 2004, 85, 684.

(10) Faÿ, S.; Feitknecht, L.; Schlüchter, R.; Kroll, U.; Vallat-Sauvain,

E.; Shah, A. Sol. Energy Mater. Sol. Cells 2006, 90, 2960.

(11) Berginski, M.; et al. J. Appl. Phys. 2007, 101, 074903.

(12) Battaglia, C.; Escarré, J.; Söderström, K.; Erni, L.; et al. Nano Lett. 2011, 11, 661.

(13) Deckman, H. W.; Wronski, C. R.; Witzke, H.; Yablonovitch, E. Appl. Phys. Lett. 1983, 42, 968.

(14) Čampa, A.; Krč, J.; Malmström, J.; Edoff, M.; Smole, F.; Topič, M. Thin Solid Films 2007, 515 (15), 5968.

(15) Zhu, J.; Hsu, C.-M.; Yu, Z.; Fan, S.; Cui, Y. Nano Lett. 2010, 10, 1979.

(16) Kelzenberg, M. D.; Boettcher, S. W.; Petykiewicz, J. A.; TurnerEvans, D. B.; Putnam, M. C.; Warren, E. L.; Spurgeon, J. M.; Briggs, R. M.; Lewis, N. S.; Atwater, H. A. Nat. Mater. 2010, 9, 239.

(17) Sakai, H.; Yoshida, T.; Hama, T.; Hichikawa, Y. Jpn. J. Appl. Phys. 1990, 29, 630.

(18) Sai, H.; Kanamori, Y.; Kondo, M.; A. Appl. Phys. Lett. 2011, 98, 113502.

(19) Python, M.; Vallat-Sauvain, E.; Bailat, J.; et al. J. Non-Cryst. Solids 2008, 354, 2258.

(20) Law, M.; Green, L. E.; Johnson, J. C.; Saykally, R.; Yang, P. Nat. Mater. 2005, 4, 455.

(21) Yamamoto, K.; Nakajima, A.; Yoshimi, M.; et al. Solar Energy 2004, 77, 939.

(22) Kroll U.; Meier J.; Fesquet, L.et al. Proceedings of the 26th European Conference on Photovoltaic Energy Conversion, Hamburg, Germany, September 5-9, 2011; p 2340, DOI: 10.4229/26thEUPVSEC2011-3BO.2.6.

(23) Aberle, A. G. Thin film solar cells. Thin Solid Films 2009, 517, 4706-4710.

(24) Meillaud, F.; Shah, A.; Droz, C.; et al. Sol. Energy Mater. Sol. Cells 2006, 90, 2952.

(25) Staebler, D. L.; Wronski, C. R. Appl. Phys. Lett. 1977, 31, 292.

(26) Benagli,S.; et al. Proceedings of the 26th European Conference on Photovoltaic Energy Conversion, Hamburg, Germany, September 5-9, 2009; p 2293, DOI: 10.4229/24thEUPVSEC2009-3BO.9.3.

(27) Boccard, M.; Cuony, P.; Despeisse, M.; et al. Sol. Energy Mater. Sol. Cells 2011, 95, 195.

(28) Kambe, M. et al. Proceedings of the 3rd World Conference on Photovoltaic Energy Conversion, Osaka, Japan, 2003.
(29) Boccard, M.; Soderstrom, T.; et al. J. Photovoltaics 2012, DOI: $10.1109 /$ JPHOTOV.2011.2180514.

(30) Ding, K.; Kirchartz, T.; Pieters, B. E.; et al. Sol. Energy Mater. Sol. Cells 2011, 95, 3318.

(31) Boccard, M.; Cuony, P.; et al. to be published in J. Photovoltaics 2012, DOI: 10.1109/JPHOTOV.2011.2179414.

(32) Escarré, J.; Söderström, K.; Haug, F.-J.; Battaglia, C.; Ballif, C. Sol. Energy Mater. Sol. Cells 2011, 95, 881.

(33) Bailat, J.; Domine, D.; Schluchter, R. et al., Conference Record of the 2006 IEEE 4th World Conference on Photovoltaic Energy Conversion, Waikoloa, Hawaii, May 7-12, 2006; IEEE: Piscataway, NJ, 2006.

(34) Battaglia, C.; Erni, L.; Boccard, M.; Barraud, L.; et al. J. Appl. Phys. 2011, 109, 114501.

(35) Dominé, D.; Buehlmann, P.; Bailat, J.; et al. Phys. Status Solidi RRL 2008, 2, 163.

(36) Buehlmann, P.; Bailat, J.; Dominé, D.; et al. Appl. Phys. Lett. 2007, 91, 143505.

(37) Cuony, P.; Marending, M.; Alexander, D. T. L.; et al. Appl. Phys. Lett. 2010, 97, 213502.

(38) Despeisse, M.; Battaglia, C.; Boccard, M.; Bugnon, G.; Charrière, M.; et al. Phys. Status Solidi A 2011, 208, 1863.

(39) Nakajima, A.; Ichikawa, M.; Sawada, T.; Yoshimi, M.; Yamamoto, K. Jpn. J. Appl. Phys. 2004, 43, 7296.

(40) Battaglia, C.; Söderström, K.; Escarré, J.; et al. Appl. Phys. Lett. 2010, 96, 213504.

(41) Aryal, M.; Buyukserin, F.; Mielczarek, K.; Zhao, X.-M.; Gao, J.; Zakhidov, A.; Hu, W. J. Vac. Sci. Technol., B 2008, 26 (6), 2562.

(42) Kim, M. S.; Kim, J.-S.; Cho, J. C.; et al. Appl. Phys. Lett. 2007, 90, 123113. 